\title{
Efecto del sulodexide en la permeabilidad vascular renal en ratas diabéticas
}

\author{
Caroline González-Serryn, David Fernándes y Yaira Mathison
}

Laboratorio de Neuroquímica Funcional, Facultad de Medicina, Escuela de Medicina

"José María Vargas", Universidad Central de Venezuela. Caracas. Venezuela.

Palabras clave: nefropatía diabética; permeabilidad vascular; glicosaminoǵlicanos; sulodexide.

Resumen. El objetivo de esta investigación fue determinar el efecto de la administración prolongada del glicosaminoglicano sulodexide (SDX) sobre la permeabilidad vascular renal en ratas diabéticas con el método de azul de Evans (AE). La diabetes tipo 1, fue inducida a ratas de la eepa Sprague-Dawley mediante la administración i.v de estreptozotocina (STZ). Los animales fueron distribuidos en tres grupos $(\mathrm{C}=$ control, STZ-SS y STZ-SDX = pre-tratados con $15 \mathrm{mg} / \mathrm{Kg}$ de SDX s.c.). Cada grupo experimental fue de 10 ratas y fueron tratados durante 90 días con sulodexide (STZ-SDX) o con el volumen correspondiente de solución salina (STZ-SS). A los 45 y 95 días de iniciado el tratamiento se determinaron la función renal y la presión arterial. Después de 3 meses a 5 ratas de cada grupo se les aplicó el método de AE y las restantes fueron sacrificadas y los riñones extraídos para el estudio histopatológico. Los resultados de función renal y presión arterial fueron superiores en el grupo STZ-SS respecto al grupo STZ-SDX y la permeabilidad vascular fue menor en el grupo STZ-SDX $(9,73 \pm 1,30 \mu \mathrm{g} \mathrm{AE} / \mathrm{g}$ de tejido) a diferencia del valor obtenido en el grupo STZ-SS $(15,55 \pm 2,15 \mu \mathrm{g}$ AE/g de tejido, $\mathrm{p}<0,01 v s$ control). Estos resultados demuestran que la administración de sulodexide mejora la función renal y protege al riñón de los cambios morfológicos de la nefropatía diabética, al disminuir la pérdida de la integridad del glicocáliz asociada al incremento de la permeabilidad endotelial glomerular lo eual se relaciona con los resultados del estudio histopatológico.

Autor de correspondencia: Caroline González-Serryn. Laboratorio de Neuroquímica Funcional, Facultad de Medicina, Escuela de Medicina "José María Vargas", Universidad Central de Venezuela. Caracas. Venezuela. Correo electrónico: serrynf@gmail.com 


\title{
Effect of sulodexide on renal vascular permeability in diabetic rats
}

Invest Clin 2018; 59 (3): 216 - 229

Key words: diabetic nephropathy; vascular permeability; glycosaminoglycans; sulodexide.

\begin{abstract}
The objective of this research was to determine the effect of prolonged administration of glycosaminoglycan sulodexide (SDX) on renal vascular permeability in diabetic rats with the method of Evans blue (EA). Type 1 diabetes, was induced to Sprague-Dawley rats by administering i.v. Streptozotocin (STZ). The animals were divided into three groups $(\mathrm{C}=$ control, STZ-SS and STZ-SDX = pre-treated with SDX $15 \mathrm{mg} / \mathrm{Kg}$ s.c). Each experimental group consisted of 10 rats and they were treated for 90 days with sulodexide (STZSDX) or the corresponding volume of saline solution (STZ-SS). At 45 and 95 days of starting treatment, renal function and blood pressure were determined. After three months, the method of EA was applied to five rats of each group, the remaining were sacrificed, and their kidneys extracted for histopathological study. The results of renal function and blood pressure were higher in the STZ-SS group compared to the STZ-SDX group and vascular permeability was lower in the STZ-SDX group $(9.73 \pm 1,30 \mu \mathrm{g} \mathrm{AE} / \mathrm{g}$ tissue) as opposed to the value obtained in the STZ-SS group $(15,55 \pm 2,15 \mu \mathrm{g} \mathrm{AE} / \mathrm{g}$ of tissue, $\mathrm{p}<0.01$ vs. control). These results demonstrate that administration of sulodexide improves renal function and protects the kidney from the morphological changes of diabetic nephropathy, since it decreases the loss of the integrity of the glycocalix associated with the increased permeability of the glomerular endothelium, which is related to the results obtained from the histopathological study.
\end{abstract}

Recibido: 27-07-2017 Aceptado: 07-06-2018

\section{INTRODUCCIÓN}

La diabetes mellitus (DM) es un trastorno metabólico crónico con etiología multifuncional, caracterizado por hiperǵlicemia crónica debida a la resistencia periférica a la insulina y/o a la alteración en la secreción de esta hormona. Este trastorno metabólico genera alteraciones en el metabolismo de carbohidratos, lípidos y proteínas, que ocasionan en un plazo variable, lesiones en el endotelio vascular que conducen a alteraciones micro vasculares que pueden ocasionar ceguera, dolor neuropático y disfunción renal (1). El endotelio es un órgano dinámico que ha sido reconocido como el regulador más importante de la homeostasis vascular y en presencia de las variaciones metabólicas exhibidas en la diabetes, puede desarrollar disfunción endotelial y como consecuencia directa de esta alteración, aumento de la permeabilidad vascular (2-4). Uno de los mecanismos implicados en el incremento de la permeabilidad vascular asociada a la diabetes, es la alteración de los glicosaminoglicanos que conforman el glicocáliz endotelial (5). Investigaciones realizadas sobre el papel del glicocáliz en el funcionamiento de la barrera glomerular, han demostrado que la restricción de la permeabilidad del glomé- 
rulo dependiente de la carga iónica para la albúmina y otras moléculas proteicas, está a cargo de esta capa compuesta por glicoproteínas y proteoglicanos como el heparán sulfato, de carǵa negativa, que le confiere las características de negatividad al endotelio, de manera que el endotelio ǵlomerular contribuye significativamente a formar la barrera a macromoléculas $(6,7)$. La disfunción renal a mediano o largo plazo conduce al desarrollo de insuficiencia renal crónica en un porcentaje elevado de los pacientes diabéticos tanto tipo 1 como tipo 2 y actualmente constituye la primera causa de insuficiencia renal crónica (IRC) en los Estados Unidos y en la mayoría de los países occidentales (8).

La disfunción endotelial asociada a la diabetes no se limita al glomérulo renal (nefropatía diabética), por lo tanto, los pacientes diabéticos presentan un alto riesgo de sufrir complicaciones cardiovasculares como cardiopatía isquémica, accidente cerebrovascular y enfermedad vascular periférica. En estos pacientes las estrategias terapéuticas se centran en el uso de aǵentes que minimicen los factores de riesgo como hipertensión o dislipidemia y que podrían tener un efecto protector sobre el endotelio $(9,10)$. Recientemente, los esfuerzos por desarrollar terapias alternativas que permitan prevenir la disfunción endotelial han resultado en la incorporación de glicosaminoǵlicanos como el sulodexide, el cual está compuesto por una mezcla de $80 \%$ de heparina y $20 \%$ de heparán sulfato y al que se le atribuye actividad antitrombótica, profibrinolítica y antiinflamatoria, y es considerado como un agente regulador del balance lipoproteico (11-14). Los hallazgos obtenidos en modelos experimentales de disfunción endotelial, han demostrado el efecto favorable del sulodexide, al disminuir de una manera significativa el número de células endoteliales descamadas en modelos de disfunción endotelial, por lo que es considerado un agente protector del endotelio, con un posible efecto preventivo del aumento de la permeabilidad endotelial asociada a la diabetes (15). Por otra par- te, a nivel renal el sulodexide previene los cambios morfológicos del glomérulo que se presentan como consecuencia del desarrollo de la diabetes (16-19). Sin embargo, en dos grandes ensayos aleatorios doble ciego, controlados con placebo, que involucraron sujetos diabéticos con micro y macroalbuminuria, el sulodexide no loǵró reducir la excreción de albúmina en orina, lo cual pone en duda el efecto renoprotector de dicho glicosaminoglicano $(20,21)$.

Debido a lo antes expuesto, hoy en día sigue siendo considerado controversial el posible efecto renoprotector del sulodexide en pacientes diabéticos, por lo que se hace imprescindible ampliar el análisis de la extravasación de proteínas. La cuantificación de la extravasación de proteínas plasmáticas mediante la determinación de los valores de proteinuria y microalbuminuria, ha permitido evaluar la disfunción endotelial asociada a alteraciones en la permeabilidad vascular a nivel glomerular. Este tipo de pruebas en modelos animales pueden ser influenciadas por varios factores que afectan la permeabilidad de la barrera endotelial, entre los que se incluyen la edad y el peso del animal, condiciones ambientales como la temperatura, la humedad, y muy importante el estrés de manipulación de los animales de experimentación. Considerando lo anteriormente descrito, se escogió el método de azul de Evans para cuantificar la extravasación de proteínas plasmáticas de forma directa. El método de azul de Evans ha permitido evaluar este parámetro gracias a la propiedad del colorante de unirse cuantitativamente a la albúmina, tanto in vitro como in vivo. Esta propiedad ha sido ampliamente usada para cuantificar la extravasación proteica como un índice del incremento de la permeabilidad vascular e indirectamente del daño tisular (22). Gracias a esta característica fue el método seleccionado en este trabajo de investigación para determinar posibles cambios en la permeabilidad endotelial en el riñón en un modelo de diabetes tipo I (DMI), luego del tratamiento con el sulodexide y 
contribuir de este modo con el estudio de los posibles efectos terapéuticos de este tipo de ǵlicosaminoglicanos en la nefropatía diabética.

\section{MATERIALES Y MÉTODOS}

\section{Animales}

Los experimentos fueron realizados en ratas de la cepa Sprague-Dawley, machos, de 8 a 10 semanas de edad, con un peso comprendido entre 250-300 g, las cuales se dividieron en los siguientes grupos $(n=10 /$ grupo): grupo control, grupo STZ-SS (diabéticas tratadas con solución salina) y grupo STZ-SDX (diabéticas tratadas con una dosis de $15 \mathrm{mg} / \mathrm{kg} /$ día de sulodexide). Todos los experimentos fueron realizados siguiendo las buenas prácticas para el manejo de animales de laboratorio y fueron aprobados por el Comité de Bioética de la Escuela de Medicina José María Vargas (22). Los animales fueron mantenidos con períodos alternos de luz y oscuridad, permitiéndoles libre acceso al agua y a la comida (comida estándar para ratas de laboratorio con un $21 \%$ de proteína, $4 \%$ de grasas, $5 \%$ de fibra y $52 \%$ de carbohidratos).

\section{Inducción de la diabetes}

La diabetes fue inducida mediante la administración de una inyección única de estreptozotocina (Sigma Chemical Co, St. Louis, MO), $60 \mathrm{mg} / \mathrm{Kg}$ de peso, en la vena caudal. El incremento de la ǵlicemia fue confirmado a $\operatorname{los} 2$ y 7 días posteriores a la administración de estreptozotocina, mediante la determinación de los niveles sanguíneos de glucosa utilizando un método enzimático (Glucosa HK Reagent, Bayer), y posterior a ello los animales fueron asignados al azar a los diferentes grupos de tratamiento. Fueron considerados animales francamente diabéticos los que tuviesen un nivel de glicemia superior a 160,20 mg/dL, según el rango considerado normal para la especie, sexo y edad (23).

\section{Tratamiento con sulodexide}

El tratamiento con sulodexide, en las ratas del grupo STZ-SDX, se inició a los 7 días posteriores a la inyección de estreptozotocina, administrándolo por vía subcutánea, a la dosis de $15 \mathrm{mg} / \mathrm{Kg} /$ día, por tres meses.

\section{Fármacos}

El sulodexide (Vessel Due ${ }^{\circledR}$ ), fue suministrado en ampollas por Laboratorios Elmor S.A. Caracas, Venezuela. Cada ampolla contiene: sulodexide $60 \mathrm{mg}$ equivalente a 600 LSU (unidad de liberación lipasémica), excipientes esp $2 \mathrm{~mL}$

\section{Determinación de las variables metabólicas}

Los niveles de ǵlicemia, triǵlicéridos en plasma, colesterol en plasma y creatinina plasmática fueron determinados mediante espectrofotometría, utilizando los siguientes estuches comerciales de Stanbio Laboratory (Texas, USA): Stanbio Glucosa Liquid Color, Stanbio Triǵlicéridos Liquid Color, Stanbio Colesterol Liquid Color y Stanbio Creatinina directa Liquid Color, respectivamente, a los 45 y 90 días de iniciado el tratamiento con sulodexide o con el volumen equivalente de solución salina.

\section{Determinación de la presión arterial}

El registro de la presión arterial, se realizó en ratas conscientes, utilizando un método no invasivo, mediante el uso de un pletismógrafo digital de cola (LE 5000, LETICA Scientific Instrument, Barcelona, España). Las ratas fueron sometidas a calentamiento para producir vasodilatación de los vasos sanguíneos periféricos colocándolas en una estufa $\left(42^{\circ} \mathrm{C}\right)$ durante 15 minutos, utilizando para ello una estufa (Memmert, 854 Schawabach, Western Germany). Posteriormente se inmovilizaron en un cepo, y se les colocó en la cola el manguito para determinar la presión arterial, este manguito estuvo conectado al transductor de pulso que permite el registro de este parámetro, midiendo además la frecuencia cardiaca (FC). Los 
valores reportados son el promedio de tres registros sucesivos, obtenidos al inicio dela etapa experimental (LB) y a los 45 días y 90 días de iniciado el experimento.

\section{Determinación de proteínas en la orina}

La determinación de las proteínas en la orina se llevó a cabo por el método de rojo de Ponceau, luego de realizar una curva estándar con albúmina sérica bovina y posterior comparación de los valores obtenidos por espectrofotometría con los resultados obtenidos de las muestras de orina, recolectadas a los 45 días y 90 días de iniciado el experimento.

\section{Cuantificación de la extravasación de proteínas plasmáticas}

La cuantificación de la extravasación de proteínas plasmáticas se realizó al finalizar la etapa experimental con el método de azul de Evans (22). Para ello, se tomaron al azar 5 animales pertenecientes a cada grupo de experimentación (control, SSTZ-SS y STZSDX) y fueron anestesiados con pentobarbital, la vena yugular fue canulada y el azul de Evans fue administrado a una concentración de $50 \mathrm{mg} / \mathrm{kg}$ de peso $(20 \mathrm{mg} / \mathrm{mL}+$ heparina $100 \mathrm{UI} / \mathrm{mL}$ ), a una velocidad de $1 \mathrm{~mL} /$ min durante 2 min. Quince minutos después, el colorante fue removido del sistema cardiovascular por perfusión intracardiaca de $25 \mathrm{~mL}$ de solución salina (NaCl 0,9\%) a $37^{\circ} \mathrm{C}$ y a una de velocidad de $25 \mathrm{~mL} / \mathrm{min} \mathrm{du}$ rante $2 \mathrm{~min}$. Posteriormente, se obtuvo una muestra del riñón izquierdo de aproximadamente $0,6 \mathrm{~g}$ de peso, la cual se colocó en un volumen exactamente medido de formamida (2 mL). El contenido del colorante azul de Evans de la muestra de riñón fue determinado después de su extracción con formamida (durante 24 horas, a $60^{\circ} \mathrm{C}$ ), utilizando espectrofotometría a $620 \mathrm{~nm}$. La cantidad de azul de Evans fue reportada en $\mu \mathrm{g} \mathrm{AE} / \mathrm{g}$ tejido.

\section{Estudios histopatológicos}

$\mathrm{Al}$ finalizar los 3 meses de tratamiento se sacrificaron al azar 5 animales pertenecientes a cada grupo y seguidamente fueron desangrados y extraídos sus riñones izquierdos. De cada riñón se cortó un polo que incluía corteza y médula y se tiñó con hematoxilinaeosina. Todos los cortes fueron examinados al microscopio óptico (aumento $250 \mathrm{X}$ ) buscando evidencias de cualquier cambio celular inducido por la DMI y el posible papel del sulodexide ante el desarrollo de las alteraciones del tejido asociadas al modelo en cuestión.

\section{Análisis Estadístico}

Se aplicó la prueba de variancia ANOVA de una vía y los resultados fueron reportados como la media \pm el error estándar de la muestra (EEM). Valores de $\mathrm{p} \leq 0,05$ se consideraron estadísticamente significativos.

\section{RESULTADOS}

\section{Valoración de las variables metabólicas}

En la Tabla I se muestran los promedios de glicemia basal y los obtenidos a los 45 y 90 días de iniciada la etapa experimental. Luego de la inducción de la DMI se elevaron los niveles de ǵlicemia en el grupo experi-

TABLA I

VALORES DE GLICEMIA

\begin{tabular}{cccc}
\hline Grupo & $\begin{array}{c}\text { Glicemia Basal } \\
(\mathrm{mg} / \mathrm{dL})\end{array}$ & $\begin{array}{c}\text { Glicemia } 45 \text { días } \\
(\mathrm{mg} / \mathrm{dL})\end{array}$ & $\begin{array}{c}\text { Glicemia } 90 \text { días } \\
(\mathrm{mg} / \mathrm{dL})\end{array}$ \\
\hline Control & $111,93 \pm 2,58$ & $120,54 \pm 5,76$ & $130,70 \pm 3,95$ \\
STZ-SS & $112,56 \pm 5,19$ & $505,14 \pm 16,09^{*}$ & $498,89 \pm 22,63^{*}$ \\
STZ-SDX & $113,57 \pm 3,18$ & $500,35 \pm 20,85^{*}$ & $466,89 \pm 27,21^{*}$ \\
\hline
\end{tabular}

Datos expresados como la media \pm error estándar, para un mínimo de 10 animales. *p $<0,001$ vs control. 
mental SDZ-SS y en el ǵrupo experimental STZ-SDX, con diferencias estadísticamente significativas respecto al grupo control, sin presentarse diferencia estadísticamente significativa entre los niveles de hiperǵlicemia de ambos grupos diabéticos a los 45 y 90 días (Tabla I)

Los niveles de triglicéridos plasmáticos y de colesterol plasmático obtenidos a lo largoo del experimento se muestran en la Tabla II. Los valores de triglicéridos del grupo control a los 45 días y 90 días de iniciado el experimento se ubicó en el rango normal. Por su parte, ambos grupos diabéticos (STZ-SS y STZ-SDX) evidenciaron un incremento considerable de los niveles de triglicéridos respecto al grupo control a los 45 días y al finalizar la etapa experimental. En cuanto a los valores de colesterol a los 45 días de tratamiento, se acercaron a los valores normales reportados para la especie en todos los grupos de experimentación. Transcurridos 90 días de iniciada la etapa experimental, el valor de colesterol del grupo control siǵuió estando dentro del rango considerado normal en ratas y en los grupos restantes hubo un ligero aumento de los valores de colesterolemia, con una diferencia estadísticamente significativa entre el subgrupo STZ-SS y el grupo control.

En relación a los valores obtenidos de creatinina plasmática mostrados en la Tabla III, los resultados del subgrupo STZ-SS a los 45 días de observación, evidenciaron un incremento estadísticamente significativo respecto al grupo control y al subgrupo STZSDX por encima de los valores considerados normales para la especie. A los 90 días de la etapa experimental los valores de creatinina plasmática se incrementaron en ambos grupos diabéticos, sin embargó, el grupo diabético STZ-SS muestra una diferencia estadísticamente significativa respecto al grupo control.

Los valores de presión arterial media (PAM), presión sistólica (PS) y presión diastólica (PD), a lo largo del tiempo se muestran en la Tabla IV. Los valores de PAM se

TABLA II

VALORES DE TRIGLICÉRIDOS Y COLESTEROL PLASMÁTICOS

\begin{tabular}{cccc}
\hline & Grupo & 45 días & 90 días \\
\hline & Control & $66,07 \pm 6,15$ & $79,30 \pm 7,96$ \\
Triglicéridos & STZ-SS & $326,87 \pm 29,18^{* *}$ & $515,79 \pm 24,38^{* *}$ \\
$(\mathrm{mg} / \mathrm{dL})$ & STZ-SDX & $257,87 \pm 28,71^{* *}$ & $460,51 \pm 31,74^{* *}$ \\
\hline & Control & $58,60 \pm 2,29$ & $0,05 \pm 1,8$ \\
Colesterol & STZ-SS & $64,72 \pm 3,92$ & $88,18 \pm 9,69^{*}$ \\
$(\mathrm{mg} / \mathrm{dL})$ & STZ-SDX & $60,36 \pm 1,18$ & $76,72 \pm 5,29$ \\
\hline
\end{tabular}

Datos expresados como la media \pm error estándar, para un mínimo de 10 animales. * ${ }^{*}$ p $<0,001$ vs control, * $\mathrm{p}<0,05$ vs control.

TABLA III

VALORES DE CREATININA PLASMÁTICA

\begin{tabular}{cccc}
\hline & Grupo & 45 días & 90 días \\
\hline & Control & $1,20 \pm 0,23$ & $1,98 \pm 0,49$ \\
Creatinina en plasma & STZ-SS & $3,21 \pm 0,57^{* *}$ & $7,29 \pm 1,51^{* *}$ \\
$(\mathrm{mg} / \mathrm{dL})$ & STZ-SDX & $1,72 \pm 0,12^{*}$ & $4,55 \pm 1,01$ \\
\hline
\end{tabular}

Datos expresados como la media \pm error estándar, para un mínimo de 10 animales. * $\mathrm{p}<0,01$ vs control, *p<0,05 al comparar el grupo STZ-SDX frente al grupo STZ-SS.

Vol. 59(3): 216 - 229, 2018 
hallaron incrementados a los 45 días y 90 días de iniciado el experimento, en relación con los valores basales en los animales francamente diabéticos, sin evidenciarse una diferencia estadísticamente significativa entre la PAM del grupo STZ-SS y la PAM del grupo STZ-SDX. Los valores de presión sistólica (PS) basal, estaban dentro del rango normal para la especie, sin presentarse diferencia estadísticamente significativa entre los grupos del ensayo. Transcurridos 45 días de tratamiento y al desarrollarse el modelo de DMI, los valores de PS se mantuvieron dentro del rango normal en el grupo control y en el grupo STZ-SDX, elevándose solo en el grupo STZ-SS. En cuanto a los valores de PD luego de 45 días de tratamiento, se mantuvieron normales en el grupo control y se elevaron en mayor proporción en el grupo diabético tratado con solución salina. Tales elevaciones se presentaron también a los 90 días de tratamiento, mostrándose nuevamente de manera más evidente en el grupo diabético tratado con solución salina.

\section{Determinaciones urinarias}

Con respecto a la proteinuria, los valores mostrados en la Tabla $\mathrm{V}$ evidenciaron que los niveles basales estaban dentro de los valores normales para la especie y que la inducción de la DMI produjo un incremento significativo de la excreción urinaria de albúmina con respecto al grupo control a los 45 días se observación en el grupo diabético tratado con solución salina. El tratamiento durante 45 días con SDX permitió prevenir el incremento en la excreción de albúmina en el grupo diabético tratado con SDX respecto al grupo diabético tratado con solución salina, lo cual fue confirmado por los resultados obtenidos a los 90 días del ensayo experimental. Por su parte, los resultados obtenidos de la extravasación de albúmina (Tabla V) al realizar el método colorimétrico azul de Evans, muestran un aumento de la extravasación de albúmina en ambos grupos diabéticos (STZ-SS y SRZ-SDX) al compararlos con los del grupo control, sin embargo, la diferencia respecto al control fue mucho más significativa en el grupo STZ-SS.

TABLA IV

VALORES DE PRESIÓN ARTERIAL

\begin{tabular}{|c|c|c|c|c|}
\hline & Grupo & Basal & 45 días & 90 días \\
\hline \multirow{3}{*}{$\begin{array}{l}\text { Presión } \\
\text { arterial } \\
\text { media } \\
(\mathrm{mmHg})\end{array}$} & Control & $101,94 \pm 4,45$ & $96,71 \pm 6,43$ & $86,67 \pm 7,90$ \\
\hline & STZ-SS & $100,71 \pm 4,45$ & $135,96 \pm 6,86^{* * *}$ & $136,55 \pm 5,97 * * *$ \\
\hline & STZ-SDX & $99,93 \pm 4,76$ & $112,08 \pm 3,13^{*}$ & $116,44 \pm 5,39^{* *}$ \\
\hline \multirow{3}{*}{$\begin{array}{l}\text { Presión } \\
\text { arterial } \\
\text { sistólica } \\
(\mathrm{mmHg})\end{array}$} & Control & $107,81 \pm 8$ & $120,14 \pm 6,91$ & $114,66 \pm 5,11$ \\
\hline & STZ-SS & $119,77 \pm 3,67$ & $156,33 \pm 6,05^{* * *}$ & $149,44 \pm 5,10^{* * *}$ \\
\hline & STZ-SDX & $117,16 \pm 3,35$ & $131,37 \pm 3,59^{*}$ & $137,11 \pm 8,7$ \\
\hline \multirow{3}{*}{$\begin{array}{c}\text { Presión } \\
\text { arterial } \\
\text { diastólica } \\
\text { (mmHg) }\end{array}$} & Control & $84,45 \pm 5,57$ & $84,71 \pm 7,73$ & $78,33 \pm 7,55$ \\
\hline & STZ-SS & $86,88 \pm 3,73$ & $127,04 \pm 7,29 * * *$ & $127,00 \pm 6,60^{* * *}$ \\
\hline & STZ-SDX & $88,33 \pm 4,63$ & $99,62 \pm 4,76^{*}$ & $100,66 \pm 6,13^{*}$ \\
\hline
\end{tabular}

Datos expresados como la media \pm error estándar, para un mínimo de 10 animales. *** $<0,001$ vs control, * $\mathrm{p}<0,05$ vs control, *p<0,05 al comparar el grupo STZ-SDX frente al grupo STZ-SS. 
TABLA V

VALORES DE ALBÚMINA EN ORINA Y EXTRAVASACIÓN DE ALBÚMINA

\begin{tabular}{|c|c|c|c|}
\hline & Grupo & 45 días & 90 días \\
\hline \multirow{3}{*}{$\begin{array}{l}\text { Albumina en orina } \\
\qquad(\mathrm{mg} / 24 \mathrm{hl})\end{array}$} & Control & $8,90 \pm 0,59$ & $8,88 \pm 0,26$ \\
\hline & STZ-SS & $24,93 \pm 2,54 * * *$ & $30,34 \pm 2,82^{* * *}$ \\
\hline & STZ-SDX & $14,86 \pm 2,58^{+}$ & $18,51 \pm 2,75^{*,++}$ \\
\hline \multirow{3}{*}{$\begin{array}{l}\text { Extravasación de albúmina } \\
\qquad(\mu \mathrm{g} \mathrm{AE} / \mathrm{g} \text { de tejido })\end{array}$} & Control & --.--------- & $6,31 \pm 0,61$ \\
\hline & STZ-SS & 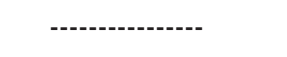 & $15,55 \pm 2,15^{* *}$ \\
\hline & STZ-SDX & --.----.- & $9,73 \pm 1,30^{+}$ \\
\hline
\end{tabular}

Datos expresados como la media \pm error estándar, para un mínimo de 10 animales. * p <0,001 vs control, "p $<0,01$ vs control, " $\mathrm{p}<0,05$ vs control, + p <0,05 al comparar el grupo STZ-SDX frente al grupo STZ-SS, ++ $<<0,01$ al comparar el grupo STZ-SDX frente al grupo STZ-SS.

\section{Estudios histopatológicos}

La evaluación de los cortes histológicos con tinción de hematoxilina-eosina de riñones del grupo control mostró la estructura normal del intersticio renal de ratas macho Sprague Dowley. En la Fig. 1 se pueden observar tres glomérulos renales viables, sin cambios segmentarios o difusos, con una matriz y celularidad mesangial conservada, paredes capilares con espesor regular y luces abiertas con espacio urinario amplio (triángulo). Los vasos sanguíneos pareeen no mostrar evidencias de necrosis o inflamación y los túbulos renales no presentan inclusiones celulares ni vacuolización y poseen membrana basal conservada. En la Fig. 2, se destacan los cambios a nivel de los glomérulos renales del grupo STZ-SS, los cuales aparentemente muestran espacios urinarios reducidos (triángulo), cambios difusos, aumento de la matriz mesangial, paredes capilares engrosadas con luces colapsadas y adherencias a la cápsula de Bowman. Los túbulos renales presentan cilindros hialinos, hemáticos, granulosos y con una degeneración hidrópica acentuada. En estos riñones se puede apreciar un posible reforzamiento fibroso de las paredes vasculares, aunque la mayor parte de los vasos presentan las luces permeables (flecha). La Fiǵ. 3, exhibe una estructura renal del grupo STZ-SDX la cual pareciera ser cercana a lo normal para la especie, con ǵlomérulos renales en su mayoría viables. Sin embargo, por efecto de la DMI, algunos muestran luces congestivas y los túbulos renales evidencian cilindros hialinos y degeneración hidrópica, en este caso pudiese sugerirse que no es tan acentuada como en las muestras de riñón de animales con diabetes tratados con solución salina.

\section{DISCUSIÓN}

La insuficiencia renal por nefropatía, es la principal causa de muerte en los pacientes con diabetes, como consecuencia del incremento de la probabilidad de desarrollar las complicaciones vasculares que caracterizan a este tipo de enfermedad metabólica $(24,25)$. Uno de los signos que se asocian al daño vascular, es el incremento de la permeabilidad endotelial la cual puede estar asociada con microalbuminuria en estadios posteriores. En la actualidad, esta se considera como una de las primeras manifestaciones de la enfermedad vascular (26). Cuando existe microalbuminuria, es porque se ha alterado de forma permanente la membrana basal glomerular, aumentando el tamaño de sus poros debido a cambios funcio- 


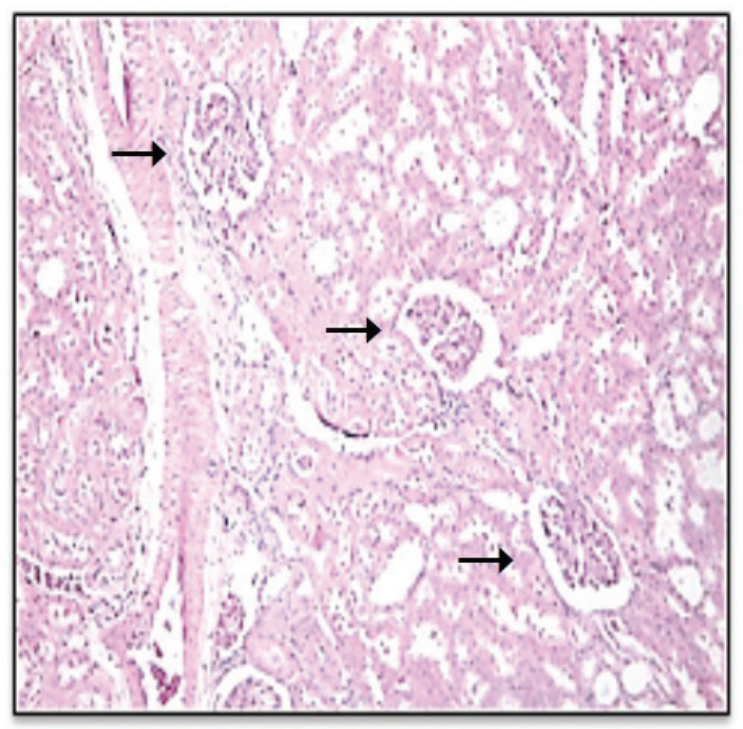

Fig. 1. Microfotografía de corteza renal de animales control donde se observan tres ǵlomérulos renales viables (flechas), sin aparentes cambios segmentarios o difusos, con una matriz y celularidad mesangial conservada, paredes capilares con espesor regular y luces abiertas con espacio urinario amplio. (250X, hematoxilina-eosina).

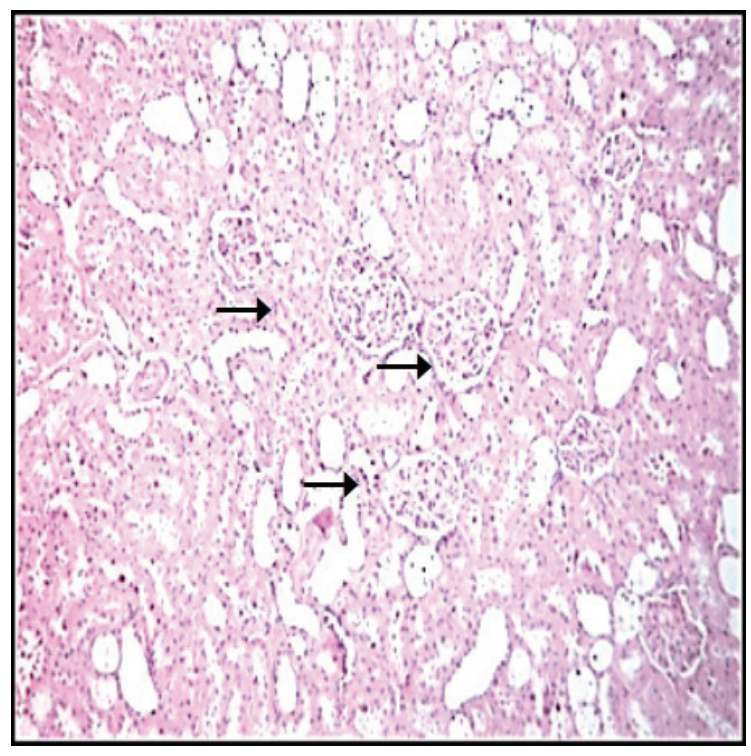

Fig. 2. Microfotografía de corteza renal de animales diabéticos tratados con solución salina en la que se observan glomérulos renales (flechas), que muestran espacios urinarios reducidos con cambios difusos, aumento de la matriz mesangial, paredes capilares engrosadas con luces colapsadas y adherencias a la cápsula de Bowman (250X, hematoxilina-eosina).

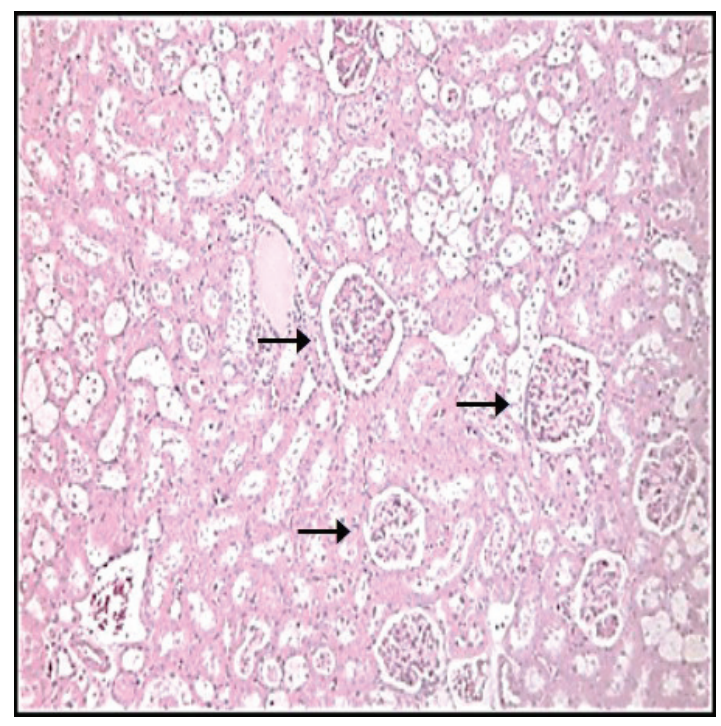

Fig. 3. Microfotografía de corteza renal de animales diabéticos tratados con sulodexide donde se observan glomérulos renales (flechas) en su mayoría con una estructura renal cercana a lo normal y sin adherencias a la capsula de Bowman. (250X, hematoxilina-eosina).

nales y estructurales como la pérdida de la selectividad de carga a nivel de la membrana basal, lo cual facilita que progrese la lesión glomerular (27-31). La patofisiología del incremento de la permeabilidad endotelial y la asociación de esta con riesgo cardiovascular permanecen aún por definirse, sin embargo, se sabe que la hiperǵlicemia es un factor causal de dichas alteraciones. En el presente estudio, se escogió la prueba de azul de Evans para cuantificar la extravasación de proteínas plasmáticas de manera directa y relacionar los resultados obtenidos con posibles cambios en las variables metabólicas, parámetros cardiovasculares, determinaciones urinarias y estudios histopatológicos, al ser tratados los animales de experimentación francamente diabéticos con sulodexide.

Con relación a las variables metabólicas y específicamente al perfil lipídico, los niveles de triglicéridos en ambos grupos diabéticos mostraron un incremento considerable respecto al grupo control a los 45 y 90 días de tratamiento. Por su parte, las 
determinaciones de colesterol plasmático a los 45 días, fueron normales en todos los grupos, sin embargo, a los 90 días de tratamiento se evidenció un ligero incremento en los grupos diabéticos, que solo fue estadísticamente significativo respecto al control en el grupo diabético tratado con solución salina. Estos resultados tienen relación con el uso inicial del sulodexide en la medicación hipolipidémica debido a su efecto sobre la liberación de lipoproteína lipasa, luego de su administración por vía parenteral u oral, lo cual se relaciona con los resultados obtenidos en un modelo experimental de hipercolesterolemia realizado en conejos, donde se demostró que el tratamiento con sulodexide reduce los niveles plasmáticos de colesterol y la acumulación del mismo a nivel de la aorta abdominal en animales con hipercolesterolemia. Del mismo modo, se confirmó el efecto hipolipidémico del sulodexide al disminuir los niveles de la lipoproteína de baja densidad (LDL) en ratas hiperlipidémicas o hipertriǵliceridémicas (32-34).

$\mathrm{Al}$ evaluar la función cardiovascular se evidenció que los resultados obtenidos de PAM, PS y PD se ajustaban a los obtenidos en modelos animales de diabetes e hipertensión, basados en el incremento de los carbohidratos en la dieta, los cuales asociaron la aparición de hipertensión con el desarrollo de insulinoresistencia (35-37). En ese sentido, se evidenció un aumento de la PAM respecto al control a los 45 días de observación en el grupo STZ-SS, con un incremento considerable en ambos grupos diabéticos a los 90 días. En relación a la PS y PD, estas se incrementaron significativamente a los 45 días y 90 días de iniciado el tratamiento en el grupo STZ-SS. El desarrollo de hipertensión a los 45 días de la etapa experimental en el grupo STZ-SS y a mayor escala a los 90 días en comparación al subgrupo STZ-SDX, puede relacionarse con el efecto de este fármaco del tipo glicosaminoglicano en la prevención de la pérdida de la respuesta vasodilatadora del endotelio, inducida por la diabetes, tal como lo demuestran los resultados obteni- dos del tratamiento con sulodexide en ratas diabéticas, sobre la relajación inducida por acetilcolina en la aorta y arteria mesentérica $(38,39)$. Adicionalmente, otras investigaciones han aportado evidencia de que la reducción en la capacidad de relajación dependiente de endotelio inducida por la diabetes, parece ser el resultado de deficiencia en la producción de óxido nítrico (ON), lo cual permite sugerir que el efecto protector sobre el endotelio ejercido por el sulodexide, está relacionado con la preservación de la acción del $\mathrm{ON}$, al incrementarse la sintasa de óxido nítrico en el riñón (40). Por otra parte, se ha demostrado que la degradación del ácido hialurónico en el ǵlicocáliz induce la pérdida de relajación ante el estrés y disminuye la liberación de $\mathrm{ON}$, indicando una relación entre las funciones mediadas por el ON y los ǵlicosaminoǵlicanos (41).

La determinación de los valores de creatinina plasmática obtenidos a los 45 días de tratamiento mostró hiperereatinemia en el grupo diabético tratado con solución salina. En este mismo periodo de observación, el valor de creatinina sérica del grupo diabético tratado con sulodexide no evidencio un aumento respecto al grupo control, lo cual permitiría inferir que la función renal comenzó a estar comprometida en el grupo diabético que no recibió sulodexide. Luego de finalizada la etapa experimental a los 90 días de tratamiento, los valores de creatinina plasmática se incrementaron en ambos grupos diabéticos. Dicho incremento fue estadísticamente significativo para el grupo diabético tratado con solución salina al compararlo con los resultados del grupo control y, adicionalmente, también mostró el nivel de creatinina sérica de este grupo una diferencia estadísticamente significativa $(p<0,05)$ con los resultados obtenidos en el grupo diabético tratado con sulodexide.

Los resultados obtenidos de la determinación de las proteínas en la orina, evidencian el incremento significativo de la excreción urinaria de albúmina únicamente en el grupo diabético tratado con solución 
salina. Se podría sugerir en consecuencia, que el tratamiento con sulodexide durante 3 meses permitió prevenir el incremento de la excreción de albúmina en los animales diabéticos. En ese sentido, relacionando los valores de albuminuria con los valores de creatinemia se confirma que el daño renal fue en cierto grado mayor en aquellas ratas que no reciben tratamiento con SDX, lo cual se relaciona con resultados obtenidos en modelos experimentales de diabetes en ratas y como se evidencia en diversos ensayos clínicos realizados en pacientes diabéticos con glomerulonefritis que fueron tratados con sulodexide, pudiendo considerarse que este efecto beneficioso sobre la integridad endotelial, podría ser eficaz en la prevención del desarrollo de otras microangiopatías en la diabetes (42-47).

A pesar de la importancia clínica de la nefropatía diabética, los mecanismos de patogénesis siguen siendo confusos. En gran parte, todavía no se ha entendido la naturaleza del aumento de la permeabilidad endotelial. El poco conocimiento que se tiene se debe a que la permeabilidad microvascular es intrínsecamente difícil de medir, tomándose como una medida de permeabilidad, el flujo de un solvente o de un soluto (coeficiente de permeabilidad de soluto, coeficiente de filtración de fluido, etc.). Dichas medidas están obstaculizadas por factores hemodinámicos como la velocidad de flujo, el gradiente de presión hidrostática, la concentración de soluto y la superficie disponible para el intercambio (48). Muy pocos estudios que describen la medida de la permeabilidad con respecto a la diabetes han controlado todos estos factores. Una de las pruebas que permite controlar en buena medida los factores hemodinámicos antes mencionados, es la prueba de azul de Evans, ampliamente utilizada para determinar la permeabilidad de vasos sanguíneos y a nivel miofibrilar en modelos in vivo e in vitro (48-52). El colorante azul de Evans (T-1824) es una molécula de 961 Da que se asocia fuertemente a la albúmina de manera irreversible. El complejo azul de
Evans-albúmina puede ser identificado por auto-fluorescencia en tejido a través de un microscopio de fluorescencia, y determinado y cuantificado por espectrofotometría de muestras de suero o de tejido homogeneizado, siendo esta ultima la técnica utilizada en nuestro estudio para cuantificar el complejo Evans-albúmina en las muestras de riñón de cada uno de los grupos de experimentación. Los resultados obtenidos de la espectrofotometría de las muestras de riñón evidenciaron una menor proporción del complejo Evansalbúmina en las muestras pertenecientes al grupo control al compararlos con los valores mostrados por los grupos diabéticos. Considerando estos resultados, como una medida de extravasación de proteínas plasmáticas, y en consecuencia de permeabilidad endotelial, a pesar de no contar con valores de referencia de otros trabajos de investigación relacionados con la permeabilidad endotelial en modelos animales de DMI basados en la prueba de Azul de Evans, se podría inferir que efectivamente el tratamiento con sulodexide permitió prevenir o disminuir el ǵrado de alteraciones en la integridad glomerular debidas a la diabetes y en consecuencia, el aumento en la permeabilidad microvaseular tal como lo evidencian diferentes ensayos clínicos $(53,54)$. Por su parte, el análisis histopatológico pudiese sugerir que el sulodexide posee un efecto protector de los glicosaminoglicanos ante los cambios morfológicos producidos por la diabetes a nivel de los glomérulos renales, ya que las alteraciones estructurales asociadas a esta patología, se evidencian en menor grado en los animales tratados con sulodexide al compararlos con los cambios en la histoarquitectura del riñón de los animales francamente diabéticos tratados con solución fisiológica. Para poder afirmar que efectivamente se previenen los cambios estructurales asociados a la diabetes en los riñones de animales diabéticos tratados con sulodexide, los hallazgos histopatológicos antes mencionados deberían ser confirmados realizando un tipo de coloración más específica como lo es el 
método de tinción de PAS (ácido peryódico schiff), el cual permite detectar polisacáridos en tejidos, tanto glucógeno como mucopolisacáridos y teñir las membranas basales.

Finalmente, estos hallazgos contribuyen al conocimiento de la patogénesis de la nefropatía diabética, lo cual es fundamental para la prevención y control de los factores que favorecen su progresión y, permite inferir que efectivamente la administración exógena de glicosaminoglicanos, pudiese aminorar el avance de la permeabilidad endotelial renal que conduce a disfunción endotelial. Al determinar la extravasación de albúmina a través del método de azul de Evans, el cual consiste en una prueba colorimétrica directa y con excelentes propiedades cualitativas, la cual está sujeta a menores factores externos que pudiesen alterar los resultados y de este modo se pueden obtener evidencias experimentales que corroboran los diferentes ensayos clínicos realizados en pacientes diabéticos tratados con sulodexide.

\section{AGRADECIMIENTOS}

Nuestro especial agradecimiento a la Dra. Teresa Gledhill, del Departamento de Anatomía Patológica del Hospital Vargas de Caracas y Profesora de la Cátedra de Histología de la Escuela de Medicina "José María Vargas" por su apoyo al realizar el estudio histopatológico de las muestras de riñón empleadas en el trabajo y al Dr. Homero Augusto Campos, Profesor Titular de la Cátedra de Farmacología de la Escuela de Medicina "José María Varǵas" quien en vida asesoró y ayudó en la realización de este proyecto de investigación.

\section{REFERENCIAS}

1. Sheetz MJ, King Gl. Molecular understanding of hyperglycemia's adverse effects for diabetic complications. JAMA 2002; 288 (20): 2579-2588.

2. Furchgott RF, Zawadzki JV. The obligatory role of endothelial cells in the relaxation of arterial smooth muscle by acetylcholine. Nature 1980; 288(5789):373-376.

3. Esper RJ. Interrogando al endotelio. Rev Argentina de Cardiología 2000; 68:430-43.

4. Vanhoutte PM. Endothelial dysfunction and aterosclerosis. Eur Hearth J 1997; 18 (SE):E19-E29.

5. Me Lellan AC, Thornalley PJ, Benn J, Sonksen PH. Glycoxalase system in clinical diabetes mellitus and correlation with diabetic complications. Clin Sci 1994; 87:2129.

6. Alder S. Structure-function relationships associated with extracellular matriz alterations in diabetic glomerulopathy. J Am Soc Nephrol 1994; 5:1165-1172.

7. Kanwar YS, Liu ZZ, Kashihara N, Wallner EI. Current status of the structural and functional basis of glomerular filtration and proteinuria. Semin Nephrol 1991; 11:390413.

8. Revisan R, Viberti G. Genetic predisposition and diabetic nephropaty. Diab Nutr Metab 1995; 8: 159-165.

9. Orence O. Nuevas direcciones en la patogénesis y tratamiento de la nefropatía diabética. Rev Venez Endocrinol Metab 2004;2 (1): 2-4.

10. Pechánová $\mathrm{O}$, Zicha $\mathrm{J}$, Paulis L, Zenebe W, Dobesová Z, Kojsová S, Jendeková L, Sládková M, Dovinová I, Simko F, Kunes J. The effect of $\mathrm{N}$-acetyleysteine and melatonin in adult spontaneously hypertensive rats with established hypertension. Eur J Pharmacol 2007; 561(1-3):129-136.

11. Kristova V, Kriska M, Babal P, Djibril M, Slamova J, Kurtansky A. Evaluation of endothelium-protective effects of drugs in experimental models of endothelial damage. Physiol Res 2000; 49:123-128.

12. Harenberg, J. Review of pharmacodynamics, pharmacokinetics, and therapeutic properties of sulodexide. Med Res Reviews 1998; 18:1-20.

13. Ofosu FA. Pharmacological actions of sulodexide. Sem Thromb Hemost 1998; 24(2):127-138.

14. Karoń J, Połubinska A, Antoniewiez A., Sumińska-Jasińska K, Bręborowiez A. AntiInflammatory effect of sulodexide during acute peritonitis in rats. Blood Purif 2007; 25 (56):510-514.

Vol. 59(3): 216 - 229, 2018 
15. Li T, Liu X, Zhao Z, Ni L, Liu C. Sulodexide recovers endothelial function through reconstructing glycocalyx in the balloon-injury rat carotid artery model. Oncotarget. 2017; 8:91350-91361.

16. Gambaro G, Cavazzana AO, Luzi P, Piccoli A, Borsatti A, Crepaldi G. Glycosaminoglycans prevent morphological renal alterations and albuminuria in diabetic rats. Kidney Int 1992; 42(2):285-291.

17. Gambaro G, Kinalska I, Oksa A, Pontuch P, Hertlova M, Olsovsky J. Oral sulodexide reduces albuminuria in microalbuminuric and macroalbuminuric type 1 and type 2 diabetic patients: the Di.NAS randomized trial. J Am Soc Nephrol 2002; 13(6):16151625.

18. Shimomura H, Spiro RG. Studies on macromolecular components of human glomerular basement membrane and alterations in diabetes. Decreased levels of heparan sulfate proteogilyean and laminin. Diabetes 1987; 36(3):374-381.

19. Wijnhoven T, Lensen JF, Rops AL, MeCarthy $\mathrm{KJ}$, van der Vlag $\mathrm{J}$, Berden $\mathrm{JH}$, van den Heuvel LP, van Kuppevelt TH. Antiproteinuric effects of glycosaminoglycanbased drugs. Curr Opin Mol Ther 2007; $9(4): 364-377$.

20. Lewis EJ, Lewis JB, Greene T, Hunsicker LG, Berl T, Pohl MA, de Zeeuw D, Heerspink HL, Rohde RD, Atkins RC, Reutens AT, Packham DK, Raz I. Sulodexide for kidney protection in type 2 diabetes patients with microalbuminuria: a randomized controlled trial. Am J Kidney Dis 2011; 58(5):729-736.

21. Packham DK, Wolfe R, Reutens AT, Berl T, Heerspink HL, Rohde R, Ivory S, Lewis J, Raz I, Wiegmann TB, Chan JC, de Zeeuw D, Lewis EJ, Atkins RC. Sulodexide fails to demonstrate renoprotection in overt type 2 diabetic nephropathy. J Am Soc Nephrol 2012; 23(1):123-130.

22. Saria A, Lundberg J. Evans blue fluorescence: quantitative and morphological evaluation of vascular permeability in animal tissues. J. Neurosei Methods 1983; 8:41-49.

23. National research council. Guide laboratory animals for the care and use of laboratory animals. 8th Edition. National Academies Press; 2011, p 1-111.
24. Tschöpe C, Reinecke A, Seidl U, Yu M, Gavriluk V, Riester U, Gohlke P, Graf K, Bader M, Hilgelfeldt U, Pesquero JB, Ritz E, Unger T. Functional, biochemical, and molecular investigations of renal kallikreinkinin system in diabetic rats. Am J Physiol 1999; 277: 2333-2340.

25. Remuzzi G, Schieppati A, Ruggenenti P.Clinical Parctice. Nephropathy in patients with type 2 diabetes. $\mathrm{N}$ Engl J Med 2002; 346 (1):1145-1151.

26. Jorde R. Ability of patients with type 1 diabetes mellitus to predict changes in $\mathrm{HbA} 1 \mathrm{c}$ levels. Diabetes Metab 1999; 25(3):241245.

27. Badimon L, Martínez-González J. Disfunción endotelial. Rev Esp Cardiol 2006; 6 (Supl A):21A-30A.

28. Brownlee M. Biochemistry and molecular cell biology of diabetic complications. Nature 2001; 414(6865):813-820.

29. Brenner BM, Bohrer MP, Baylis C, Deen WM. Determinants of glomerular permselectivity: insights derived from observations in vivo. Kidney Int 1997; 12(4):229-232.

30. Lemley KV, Blouch K, Abdullah I. Glomerular permeability selectivity at the onset of nephropathy in type 2 diabetes mellitus. $J$ Am Soc Nephrol 2000; 11(11):2095-2105.

31. Benard B, Daniel Ch, Mathias J. Endothelial glycocalyx and coronary vascular permeability: the fringe benefit. Basic Res Cardiol 2010; 105(6):687-701.

32. Kristova V, Liskova S, Sotnikova R, Votjo R, Kurtansky A. Sulodexide improves endothelial dysfunction in streptozotosininduced diabetes in rats. Physiol Rev 2008; $57(3): 491-494$.

33. Radhakrishnamurthy B, Sharma C, Bhandaru RR, Berenson GS, Stanzani L, Mastacchi R. Studies of chemical and biologic properties of a fraction of sulodexide, a heparin-like ǵlycosaminoǵlycan. Atherosclerosis 1986; 60(2):141-149.

34. Cristofori M, Mastacchi R, Barbanti M, Sarret M. Pharmacokinetics and distribution of a fluoresceinated glycosaminoglycan, sulodexide, in rats. Part I: Pharmacokinetics in rats. Arzneimittelforschung 1985; 35(10):1513-1516.

35. Howard B, Mitch W, Smith S, James R, Benjamin I, Burke G, Chait A, Eckel R. 
Diabetes and cardiovascular disease: a statement for healtheare professionals from the American Heart Association. Circulation 1999; 100:1134-1147.

36. Verma S, Bhanot S, MeNeill JH. Effect of chronic endothelin blockade in hyperinsulinemic hypertensive rats. Am J Physiol 1995; 269(6): H2017- H2021.

37. Mohammed HA, Munavvar AS, Edward $\mathbf{J J}$. The relation between fructose-induced metabolic syndrome and altered renal haemodynamic and excretory function in the rat. Int J Nephrol 2011; Vol. 2011.

38. Kristova V, Liskova S, Sotnikova R, Vojtko R, Kurtansky A. Sulodexide improves endothelial dysfunction in streptozotocininduced diabetes in rats. Physiol Res 2008; 57:491-494.

39. Mathison Y, Garrido M, Israel A, Quero Z, Fernández H. Efecto del sulodexide sobre la actividad de la sintasa de óxido nítrico en la corteza renal de ratas con diabetes tipo 1. Rev Lat Hipert 2008; 3(6): 182-188.

40. Vásquez J, Mathison Y, Romero-Vecchione E y Suárez C. Efecto del sulodexide sobre la capacidad de relajación y alteraciones estructurales de la arteria aorta en ratas diabéticas por estreptozotocina. Invest Clin 2010; 51(4): 467 - 477.

41. Mochisuki S, Vink H, Hiramatsu O, Kajita T, Shigeto F, Spaan J, Kajiya F. Role of hyaluronic acid ǵlicosaminoglyeans in shearinduced endothelium-derived nitric oxide release. Am J Physiol Heart Cire Physiol 2003; 285:H722- 726.

42. Ceol M, Gambaro G, Sauer U. Glycosaminoglycan therapy prevents TGF- $\beta 1$ overexpression and pathologic changes in renal tissue of long-term diabetic rats. J Am Soc Nephrol 2009; (11): 2324-2336.

43. Dedov I, Shestakova M, Vorontzov A. A randomized, controlled study of sulodexide therapy for the treatment of diabetic nephropathy. Nephrol Dial Transplant 1997; 12(11): 2295-2300.

44. Gambaro G, Venturini A, Noonam D, Fries W, Re G, Garbisa S. Treatment with a glycosaminoglycans formulation ameliorates experimental diabetic nephropaty. Kidney Int 1994; 46:797-806.

45. Rachel MP, Steven JH, David OB. A role for the endothelial glycocalyx in regulating mi- crovascular permeability in diabetes mellitus. Cell Biochem Biophys 2007; 49: 65-72.

46. Zilişteanu D, Attasie T, Voiculeseu M. Efficacy of long-term low-dose sulodexide in diabetic and non-diabetic nephropathies. Rom J Intern Med 2015; 53(2):161-169.

47. Olde Engberink R, Vogt L. The renoprotective effects of sulodexide. Drug Des Devel Ther 2016; (10):1233-1234.

48. Yuan S, Rigor R. Regulation of endothelial barrier function. University of California Davis. San Rafael (CA) Morgan \& Claypool Life Sciences; 2010, p 10-16.

49. Hamer P, Me Geachie J, Davies M, Grounds M. Evans blue dye as an in vivo marker of myofibre damage: optimising parameters for detecting initial myofibre membrane permeability. J Anat 2002; 200(1): 69-79.

50. Ma N, Hunt N. Correlation between enhanced vascular permeability, up regulation of cellular adhesion molecules and monocyte adhesion to the endothelium in the retina during the development of fatal murine cerebral malaria. Am J Pathol 1996; 149(5):1745-1762.

51. Dodd OJ, Hristopoulos M, Scharfstein D, Brower R, Hassoun P, King LS, Becker P, Liu M, Wang W,Hassoun HT, Rabb H. Interactive effects of mechanical ventilation and kidney health on lung function in an in vivo mouse model. Am J Physiol Lung Cell Mol Physiol 2009; 296(1):L3-L11.

52. Jü-qin H, Qing-luan L, Huai You W. Spectroscopic study of the competitive interaction between streptomycin and Evans blue to bovine serum albumin. Spectrochim Acta A Mol Biomol Spectrose 2011; 83(1):28-33.

53. Broekhuizen L, Lemkes B, Mooij H, Meuwese M, Verberne H, Holleman F, Schlingemann R, Nieuwdorp M, Stroes E, Vink $H$. Effect of sulodexide on endothelial glycocalyx and vascular permeability in patients with type 2 diabetes mellitus. Diabetologia 2010; 53(12):2646-2655.

54. Li R, Xing $J$, Mu $X$, Wang $H$, Zhang $L$, Zhai Y, Zhang Y. Sulodexide therapy for the treatment of diabetic nephropathy, a metaanalysis and literature review. Drug Des Devel Ther. 2015; 9:6275-6283. 